\title{
LITHIUM ABUNDANCES OF SOLAR-TYPE STARS: A CRITICAL APPLICATION OF HIGH SIGNAL-TO-NOISE, HIGH RESOLUTION SPECTROSCOPY
}

\author{
David R. Soderblom \\ Space Telescope Science Institute \\ 3700 San Martin Drive \\ Baltimore, Maryland 21218 USA
}

\begin{abstract}
The study of lithium depletion in solar-type stars should help elucidate essential properties of convection in those objects. Recent high-quality observations have revealed extensive flaws in our understanding of this phenomenon. The state of our knowledge of $\mathrm{Li}$ depletion is reviewed, with a brief possible explanation of it all.
\end{abstract}

\section{NEW LIGHT ON OLD PROBLEMS}

The advent of highly efficient detectors capable of producing high quality stellar spectra has enabled more detailed study of phenomena that were limited by photographic data. One of these problems is the study of lithium depletion in solar-type stars.

Observationally, one is faced with the difficulty of having only one feature to measure. This is blended with a nearby $(\Delta \lambda=0.3 \AA)$, weak Fe I line, $\left(W_{\lambda}(\odot)=8.4 \mathrm{~m} \AA\right.$ ), and the $\mathrm{Li}$ line at $6708 \AA$ is frequently very weak itself (about $2 \mathrm{~mA}$ for the Sun). In stars cooler than the Sun, many weak CN lines add to the confusion. The benefit gained from high resolution and signal-to-noise is demonstrated by the many papers on $\mathrm{Li}$ abundances presented at this conference.

Having obtained a good spectrum, one wishes to analyze it to derive the star's $\mathrm{Li}$ abundance, $\log N(\mathrm{Li})$. This analysis is very temperature sensitive: an uncertainty in $T_{\text {eff }}$ of $100 \mathrm{~K}$ leads to an uncertainty in the $\mathrm{Li}$ abundance of $\pm 40 \%$. This is because most of the star's $\mathrm{Li}$ is ionized, and cannot be observed directly.

Finally, one wishes to interpret the $\mathrm{Li}$ abundance to say something about the star. We are still uncertain how good $\mathrm{Li}$ is as an age indicator (Soderblom 1984), but even if a tight relationship is assumed, the time scale for $\mathrm{Li}$ depletion is exquisitely mass sensitive. An example is $\alpha$ Cen A, which is nearly indistinguishable from the Sun spectroscopically, is only slightly more massive, yet has a substantially greater $\mathrm{Li}$ abundance (Soderblom and Dravins 1984).

Despite all this, the $\mathrm{Li}$ problem is pursued because these observations should reveal essential properties of convection. The presence of a convective envelope is the distinguishing characteristic of a late-type star. Better knowledge 
of convection is necessary to better understand other related phenomena such as magnetic dynamos and their manifestations.

The photographic data were just uncertain enough to be consistent with a nice, simple picture: stars (at least those of the young disk) are formed with the same initial $\mathrm{Li}$ abundance. This is gradually depleted over the star's main sequence lifetime, at a rate determined mainly by a star's mass, as convection carries surface $\mathrm{Li}$ to a depth where the temperatures are high enough to destroy it $(T \gtrsim 2.4 \mathrm{MK})$.

The details in this picture were poorly understood, but it made sense qualitatively. New, high-quality data are improving this picture, adding some embarassments, but also clarifying other areas.

\section{WHAT DO WE REALLY KNOW?}

(1) Over at least the past $\sim 5 \mathrm{Gyr}$, stars have formed with the same $\mathrm{Li}$ abundance, $\log N(\mathrm{Li}) \approx 3.0$. This is substantiated by the $\mathrm{Li}$ abundances of carbonaceous chondrites, $\mathrm{T}$ Tauris, and the most Li-abundant stars in young clusters (see, e.g., Boesgaard, Budge, and Burck 1988). If the interstellar medium is being further enriched in $\mathrm{Li}$, it is at a slow rate. The precise range in $\mathrm{Li}$ abundance seen in different parts of our Galaxy is unknown.

(2) The Sun has $\log N(\mathrm{Li}) \approx 1.0$ (Müller, Peytremann, and de la Reza 1975 ), about $1 \%$ of its original value. Note that these first two points provide unambiguous evidence that the Sun has depleted most of its initial $\mathrm{Li}$ (as opposed to having formed with low $\mathrm{Li}$ ).

(3) For homogeneous groups of stars (same age and composition), $\mathrm{Li}$ is strongly mass-related. There is a precipitous dip among the mid-F dwarfs now so well delineated by Boesgaard and her co-workers (e.g., Boesgaard and Tripicco $1986 a, b)$, and a more gradual decline from about GOV to lower masses (Cayrel et al. 1984).

(4) Even within such a homogeneous group there is substantial star-tostar scatter that is real, and which does not go away with advancing age. For example, see the observations of NGC 752 or M 67 of Hobbs and Pilachowski $(1986 a, b)$.

(5) Metal-poor stars (old disk and Population II) lose their Li either very slowly or not at all. Very metal-deficient stars $([\mathrm{Fe} / \mathrm{H}] \lesssim-1)$ have strikingly high abundances of $\mathrm{Li}$ (Hobbs and Duncan 1987), yet even mildly metal-deficient stars that are unarguably old (for example, $\beta$ Hyi [Rebolo et al. 1986]) have embarassingly large amounts of $\mathrm{Li}$.

(6) There is no evidence for any star having ${ }^{6} \mathrm{Li} /{ }^{7} \mathrm{Li}$ significantly greater than the solar value, despite what was thought from the photographic data (Andersen, Gustafsson, and Lambert 1984; Soderblom 1985; Rebolo et al. 1986).

\section{WHAT DO WE THINK WE KNOW?}

(1) $\mathrm{Li}$ depletion is related to convection, at least for stars at or below the sun's mass. This is strongly indicated by the more rapid depletion seen at lower masses, but other scenarios are possible. For example, one could postulate that the observed decline of $\mathrm{Li}$ with age reflects main sequence mass loss, and that that mass loss is greater in lower mass stars. (Mass loss would carry away the most. Li-rich material at the surface, leading to dilution of the remainder. 
(2) All stars in a cluster start with the same Li abundance. It seems highly unlikely that an appreciable spread would exist, especially since none is seen in $[\mathrm{Fe} / \mathrm{H}]$, but it's not impossible, particularly if the sites of $\mathrm{Li}$ formation are within star-forming regions.

(3) The $\mathrm{Li}$ depletion rate is fixed by mass, composition, and age. If stochastic factors lead to appreciable star-to-star differences over time scales of billions of years, that would seem to violate the Vogt-Russell theorem. Although differences in a star's rotation rate could influence $\mathrm{Li}$ depletion (because magnetic fields can inhibit convection), for solar-type stars there appears to be a rapid convergence to a fixed value of rotation for stars of the same age and mass. Also, it doesn't seem right that modest differences in star's initial rotation rates should have observable manifestations nearly $10 \mathrm{Gyr}$ later. It is also frustrating to see a solar temperature star in M 67 (a cluster of about the same age and composition as the Sun) that has much more $\mathrm{Li}$ than the Sun (Hobbs and Pilachowski 1986b).

(4) Chromospheric activity doesn't much affect the apparent $\mathrm{Li}$ abundance. Some of the star-to-star differences in $\mathrm{Li}$ could be explained by the influences of chromospheric activity on line formation. Both Giampapa (1986) and Boesgaard (this conference) have followed several very active stars over several rotation periods, and see no variation in the $\mathrm{Li}$ line at a level well below $1 \%$. This suggests that chromospheric activity does not strongly affect the apparent $\mathrm{Li}$ abundance of a star, although it is still possible that overall chromospheric activity levels play a role.

(5) The $\mathrm{Li}$ abundances of halo stars $(\log N(\mathrm{Li}) \approx 2.0)$ represents the $\mathrm{Li}$ created in the Big Bang. There could well have been some depletion among these stars-perhaps 3.0 is the true Big Bang value.

\section{WHAT DO WE NOT KNOW?}

(1) The mechanism of $\mathrm{Li}$ depletion. However, improved observations have spurred efforts in this area (more below).

(2) The true dependence of depletion rate on mass, composition, and ageonly better observations can help here.

not.

(3) If stochastic factors play a significant role in $\mathrm{Li}$ depletion. Let's hope

(4) If autogenesis is important in determining the observed $\mathrm{Li}$ abundances.

It's probably not, and tends to produce too much ${ }^{6} \mathrm{Li}$ anyway.

(5) If stars in clusters are really the same age.

\section{WHAT DO WE NEED TO KNOW?}

(1) $\mathrm{Li}$ in more clusters of different ages (e.g., $\alpha$ Per and the Ursa Major Group. Such observations are in press.

(2) $\mathrm{Li}$ in clusters of the same or similar age, especially if they have different compositions. For example, Praesepe and Coma should be compared in detail to the Hyades.

(3) A detailed $L i$ survey of $F, G$, and $K$ dwarfs at high resolution and signal-to-noise. Is $\mathrm{Li}$ as rare as we think it is in stars less massive than the Sun? The distributions of $\mathrm{Li}$ abundances as a function of spectral type would also provide useful information.

(4) Uncertainties in $\mathrm{Li}$ abundances. High quality data demand high quality analyses, including good quantitative estimates of uncertainties. This is partic- 
ularly important in analyzing differential effects, and for testing models against observations. Spectrum synthesis is arguably the best technique for extracting information from high quality spectra, but has not been used enough.

(5) If stars in visual binaries have consistent $\mathrm{Li}$ abundances.

(6) The trends in $\mathrm{Li}$ abundance among metal-poor stars. Although many such stars have abundant $\mathrm{Li}$, not all of them do. Two notable exceptions are $\mu$ Cas and $\tau$ Cet. This gets back to the question of just what stellar parameters determine $\mathrm{Li}$ depletion.

\section{MAKING SENSE OF IT ALL}

Some recent calculations by Stringfellow, Faulkner, and Bodenheimer (1987) suggest a way of accounting for much of what's observed. Their work will appear separately; for these purposes it is sufficient to note that their results suggest that $\mathrm{Li}$ depletion in a star or cluster is painfully sensitive to metallicity. They were able to reproduce the $\mathrm{Li}$ vs. mass (or color) curve of Cayrel et al. (1984), but found that changes in $[\mathrm{Fe} / \mathrm{H}]$ of only 10 to $20 \%$ produced very different curves.

If one were sufficiently confident of these models, they would enable the determination of cluster abundances to very high precision. More realistically, since depletion time scales are so dependent on metallicity in their models, it is probable that the internal spread in $\mathrm{Li}$ seen in clusters is due to different times of formation of individual stars. If this spread were due to star-to-star differences in $[\mathrm{Fe} / \mathrm{H}]$, the spread would grow with age, which is not seen. An exponential process (which $\mathrm{Li}$ depletion is if a constant fraction is carried to a sufficiently high temperature in a given time) preserves any initial spread in $\mathrm{Li}$, and that agrees with observation.

Their model also accounts for the substantial $\mathrm{Li}$ seen in mildly metaldeficient stars (Duncan 1981; Soderblom 1983). However, they would predict significant differences in $\mathrm{Li}$ between Hyades and the Coma cluster (same age but different metallicity), which isn't seen (Soderblom et al. 1987).

Better quality observations of stars in clusters should help delineate the trends well enough to make reasonable sense of $\mathrm{Li}$ depletion. One lesson from this, though, is reinforced by other high signal-to-noise studies: A full and accurate analysis of the observations requires a comparable level of knowledge of the relevant fundamental stellar parameters (mass, compositions, age, etc.). In fact, these parameters are generally known very poorly.

I remain convinced that we can understand the $\mathrm{Li}$ abundances of solartype stars in a reasonably straightforward fashion. There is some equation that expresses how the instantaneous $\mathrm{Li}$ depletion rate of a star depends on its overall properties, from which one could calculate its $\mathrm{Li}$ abundance history. We are getting clues to the relative weightings of various coefficients, but are a long way from pinning down values. We are not even certain what all the relevant parameters are.

\section{REFERENCES}

Andersen, J., Gustafsson, B., and Lambert, D. L. 1984, Astr. Ap., 136, 65. Boesgaard, A. M., Budge, K. G., and Burck, E. E. 1988, Ap. J., 325, in press. Boesgaard, A. M., and Tripicco, M. J. 1986a, Ap. J. (Letters), 302, L49. . 1986b, Ap. J., 303, 724. 
Cayrel, R., Cayrel de Strobel, G., Campbell, B., and Däppen, W. 1984, Ap. J., 283, 205.

Duncan, D. K. 1981, Ap. J., 248, 651.

Giampapa, M. S. 1986, private communication.

Hobbs, L. M., and Duncan, D. K. 1987, Ap. J., 317, 796.

Hobbs, L. M., and Pilachowski, C. 1986a, Ap. J. (Letters), 309, L17. . 1986b, Ap. J (Letters), 311, L37.

Müller, E. A., Peytremann, E., and de la Reza, R. 1975, Solar Phys., 41, 53.

Rebolo, R., Crivellari, L., Castelli, F., Foing, B., and Beckman, J. E., 1986, Astr. Ap., 166, 195.

Soderblom, D. R. 1983, Ap. J. Suppl., 53, 1. 1984, in Cool Stars, Stellar Systems and the Sun, eds. S. L.

Baliunas and L. W. Hartmann (Heidelberg: Springer), p. 205. 1985 Pub. A.S.P., 97, 54.

Soderblom, D. R., and Dravins, D. 1984, Astr. Ap., 140, 427.

Soderblom, D. R., Oey, M. S., Stone, R. P. S., and Johnson, D. R. H. 1987, Ap. $J .$, in preparation.

Stringfellow, G., Faulkner, J., and Bodenheimer, P. 1987, private communication. 


\section{DIscussion}

R. CAYREL In connection with the reported absence of modulation of the Lithium resonance line in active stars, I want to make the following remark :

The lithium line is enhanced in spots but weakened in plages. A simple computation shows that if the area ratio of plages/spots is as in the active sun the expected modulation is almost nil.

SODERBLOM I agree that the sense of the modulation is not obvious, which is why simultaneous data for both Li and Ca II $\mathrm{H}$ and $\mathrm{K}$ or Ha must be obtained.

REBOLO Could you give us information about the type of the stars in which rotational modulation of the lithium line has been searched and the precision obtained in the work?

SODERBLOM see the remark by Ann Boesgaard. She reports that the equivalent oridth of the lithium feature in $\chi^{1}$ Orionis changed by less than $\approx 1 / 28$ over its rotation period.

BOESGAARD I have made observations to look for rotational modulation of $\mathrm{Li}$ in 6 stars at CFFr for 4 nights. The stars included $X^{1}$ ori, $k$ Cet and others. (I can give you the list.). None showed any variation in the $L i$ equivalent width to better than 0.5 percent. In $X^{1}$ ori the LiI line is $103 \mathrm{~m}$ is $0.5 \mathrm{~m}$ i over the four nights.

SODERBLOM Did you have any measurement of the chromospheric

variations then?

BOESGAARD Yes. I asked for simultaneous observations of CarI at wt. Wilson, they had poor weather and some equipment problems. I made observations at $\mathrm{H} \alpha$ in between the $\mathrm{Li}$ observations as a check on the chromospheric activity changes. I did find some modulation in the core of Ha for $x^{1}$ Ori.

[see separate page for my other comment which followed this].

SODERBLOM This is very encouraging - at least these stars are consistently fooling us, if they're fooling us at all. I still wonder if the overall enhancement of chromospheric activity in young stars can influence the apparent $\mathrm{Li}$ abundance, but this lack of modulation to such a fine level makes that highly unlikely.

I also thank G.Marcy for clarifying a rumor that some pleiades had exhibited considerable Li variability over long time spans. They do not, which is also encouraging.

BOESGAARD You pointed the difficulties with the features which blend with the weak solar LiI line, the $\mathrm{CN}$ and the FeI line. These are not a problem for the early $F$ stars where the PeI line at $6707.441 \mathrm{~A}$ is 4 $1 \mathrm{~m}$ and $\mathrm{CN}$ is virtually non-existent.

SODERBLOM You are correct, of course- the problem is simpler until one reaches the stars where rotation blends the lines. 